\title{
On the Application of Neutron Small Angle Scattering to the Study of Fluctuations Near the Liquid-liquid Critical Point in Binary Mixtures
}

\author{
P. Damay \\ Laboratoire de Chimie Physique, CNRS, LA253, 13 rue de Toul, 59046 Lille \\ P. Chieux \\ Institut Laue Langevin 156X, 38042 Grenoble Cedex
}

Z. Naturforsch. 34a, 804-809 (1979); received April 12, 1979

\begin{abstract}
A comparative survey is made of the neutron and light scattering techniques as applied to structural investigations in the vicinity of critical points in fluids. For neutron scattering as applied to fluctuaticns in binary mixtures, some criteria for a good contrast in order to optimize the scattering parameters such as the isotopic composition or the concentration are considered. This leads us to show how to write the partial structure factors in binary mixtures in order to remove the coupling between concentration and density fluctuations artificially introduced by Bhatia and Thornton when the components are of different size. Finally, the amplitudes of the concentration fluctuations near a critical point are discussed.
\end{abstract}

It is clear, as compared to light scattering, that very little use of neutron scattering has been made up to now for the investigation of critical phenomena in fluids. Although the two approaches are, in principle, equivalent, the neutron scattering technique presents some interesting advantages which we shall emphasize while restricting ourselves to structural problems. We will then investigate in more detail the case of the binary mixtures near their liquid-liquid critical point and show what are the criteria for good contrast. These citeria are fundamental if one wishes to optimize the scattering parameters such as the isotopic composition. This will lead us to show how to express the partial structure factors in order to get rid of the coupling between concentration fluctuations and density fluctuations introduced artificially by the size difference of the two components of a binary mixture. This might bring up important simplifications in the partial structure factor evaluation of binary alloys. Finally, we shall discuss the amplitudes of the concentration fluctuations in the vicinity of a critical point.

I. General comments on the neutron seattering investigation of eritical phenomena in fluids

When speaking about critical phenomena we think about long range fluctuations in real space,

Reprint requests to $\mathrm{P}$. Chieux, Institut Laue Langevin $156 \mathrm{X}, 38042$ Grenoble Cedex, Frankreich.

$0340-4811 / 79 / 0700-0804 \$ 01.00 / 0$ therefore the scattering signal corresponding to these fluctuations will be observed only at very small values of the momentum transfer $k$ between the incoming neutron particles and the system. Since $k=4 \pi / \lambda \sin \theta$, with $2 \theta$ the scattering angle and $\lambda$ the neutron wavelength, we should either work at very small scattering angles or/and at long wavelengths. This is achieved in practice with small angle neutron scattering machines such as D 11 [1] and D 17 at the Institut Laue-Langevin (Grenoble) where $5 \lesssim \lambda \lesssim 18 \AA$ and $3 \cdot 10^{-4} \AA^{-1}<k<0.6 \AA^{-1}$. This $k$-range is rather quickly covered by the use of the multidetector technique [1].

As compared to light scattering, the large $k$-range available in the neutron experiment as combined with the rather low values of the correlation length for fluctuations which are detectable (typical values for the correlation length $\xi$ are $5 \AA \lesssim \xi \lesssim 3000 \AA$ ) allows a quite large portion of the $k, \xi$ space to be explored. Figure 1 displays the $k, \xi$ diagram [2]. The shaded areas represent the conditions of the neutron scattering experiments, the continuous line rectangle represents static light scattering conditions, the dashed line rectangle, dynamical light scattering. It is clear that neutron scattering is more appropriate to studies in critical conditions, i.e. with $k \xi \gg 1$ (upper left). The light scattering experiments, on the other hand, cover more extensively the hydrodynamics regime, $k \xi \ll 1$, (lower right). This is even more evident when one realizes that the temperature stability conditions necessary to obtain $\xi$ values greater than $10^{3}$ are 


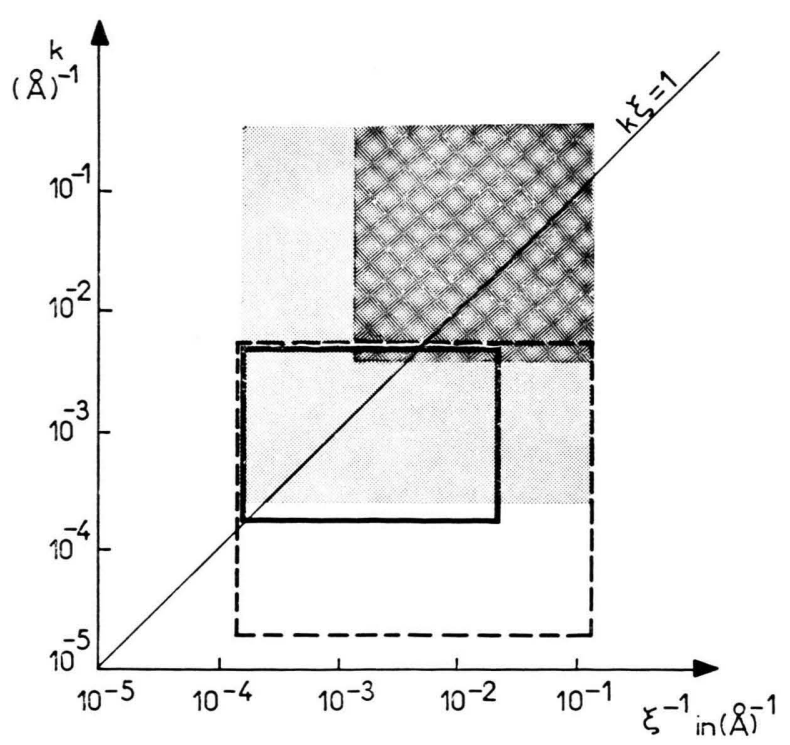

Fig. 1. The figure represents the $k \xi$ conditions of the neutron scattering experiments as compared to light scattering. The dark shaded area represents the condition of neutron scattering easily accessible experimentally (the light shaded area is technically more difficult to be achieved). The rectangle delimited by a continuous line represents static light scattering conditions, the rectangle delimited by a dashed line, dynamical light scattering. As seen light scattering covers more extensively the hydrodynamics $(k \xi \ll 1)$ conditions.

quite difficult to achieve $\left(\left(T-T_{\mathrm{c}}\right) / T_{\mathrm{c}} \lesssim 10^{-5}\right)$. Moreover, density or concentration fluctuations of correlation lengths as small as $\mathbf{5} \AA$, i.e. quite away from critical points, will easily be detected by small angle neutron diffraction. A structural basis could therefore be given to the anomalies observed in dielectric properties [3] or electronic transport properties [4], [5] quite away from the critical point in liquid binary mixtures.

On the other hand, the $k a \ll 1$ condition (where $a$ is a characteristic length of the system) or the equivalent requirement that the small angle scattering should not be perturbed by short range order effects (or near neighbors ordering) is also generally satisfied (see Figure 2). As long as we keep $k$ below 0.3 to $0.5 \AA^{-1}$ there is no interference from structural features displayed in conventional scattering experiments or structure factor determinations. The use of large wavelengths also reduces the multiple scattering problems, the main peak in the structure factor being outside the $k$-range (see Figure 2 ).

On the experimental side there are also a few unique features. Of course there is the general characteristic of the neutron beam not to be limited by the opacity of the samples and their containers or furnace assemblies but only by the absorption cross sections of the materials in the beam. This is a very favourable aspect for high temperature studies of corrosive materials [6]. It is however sometimes difficult [6] to find container materials which do not scatter at small angles, and the rather large area of the beam (about $1 \mathrm{~cm}^{2}$ which might be reduced with a corresponding loss of intensity to a beam height of $1 \mathrm{~mm}$ ) is a difficulty for gravity effects in liquid-gas critical investigation. One of the main advantages, however, as compared to light scattering is the very low background level (no parasitic effect due to the main beam) and its small $k$-dependency [7], [8] which makes it quite easy to scan the scattering signal as a function of angle or $k$.

Most of the above characteristics of neutron small angle scattering have already been applied to a few experimental cases. The good accuracy obtainable in the intensity versus $k$ plots as combined with the easiness to be in the critical conditions have been used to attempt a direct measurement of the very small critical exponent $\eta$ [9]. Difficult experimental conditions such as the study of reactive or corrosive materials, including high temperature studies have been realized $[7,10$, $11,12]$ in the investigation of systems displaying a non-metal to metal transition in the vicinity of a liquid-liquid critical point. We must recognize, however, that X-Ray small angle scattering, except for the severe difficulties related to the sample containment and the shorter $k$-range accessible in practice $\left(3 \cdot 10^{-2} \lesssim k \lesssim 0.3 \AA^{-1}\right)$ might also be applied to similar problems [13]. The uniqueness of

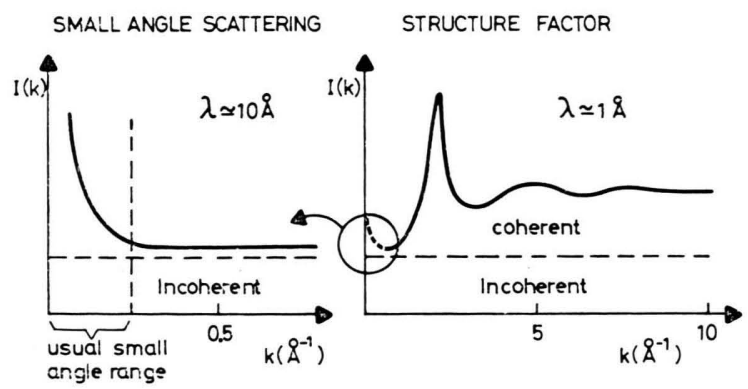

Fig. 2. The figure compares small angle neutron scattering and typical structure factor determination. It emphasizes the effects of wavelength change as well as of different geometrical conditions. The intensity scale is identical in the two pictures, the height of the small angle signal observed in the right hand side picture is limited by the lowest $\mathrm{k}$ accessible on a conventional diffractometer. 
neutron scattering becomes then quite apparent when one explores spin fluctuations such as in the recent studies on iron and cobalt far above their Curie point, up to the molten state [14]. Moreover, as we shall show below, the isotopic substitution of one element in a binary mixture might significantly alter the contrast and enhance the small angle scattering signal or eventually suppress completely one of the scattering terms.

\section{The case of binary mixtures}

In the case of binary liquid mixtures, following the Bhatia and Thornton formalism [19], the coherent neutron signal is the weighted sum of three partial structure factors.

$$
\begin{aligned}
\frac{1}{N}\left(\frac{\mathrm{d} \sigma}{\mathrm{d} \Omega}\right)^{\text {coherent }}= & \langle b\rangle^{2} S_{N N}(k)+\langle\Delta b\rangle^{2} S_{c c}(k) \\
& +2\langle b\rangle\langle\Delta b\rangle S_{N c}(k)
\end{aligned}
$$

where

$$
\langle b\rangle=c_{1} b_{1}+c_{2} b_{2},\langle\Delta b\rangle=b_{1}-b_{2} .
$$

$c_{1}, c_{2}$ and $b_{1}, b_{2}$ are the concentrations and scattering lengths of the two constituants $\left(c_{1}+c_{2}=1\right)$.

In the $k=0$ limit, following Bhatia and Thornton these three partial structure factors reach their thermodynamic limit, i.e. (see comments on these equations in Section III)

$$
\begin{aligned}
S_{N N}(0) & =\frac{N}{V} k_{\mathrm{B}} T \cdot K_{T}+\delta^{2} S_{c c}(0), \\
S_{c c}(0) & =\frac{N k_{\mathrm{B}} T}{\left(\partial^{2} G / \partial c_{2}{ }^{2}\right)_{T P N}} \\
S_{N c}(0) & =-\delta S_{c c}(0)
\end{aligned}
$$

where $N$ and $V$ are the total number of atoms and the volume of the irradiated sample.

$K_{T}$ is the isothermal compressibility.

$$
\delta=\frac{N}{V}\left(\bar{V}_{1}-\bar{V}_{2}\right) \text { with } \bar{V}_{1}, \bar{V}_{2} \text { the partial molar }
$$

volume of the two constituants. $G$ is the Gibbs free energy of the system.

$S_{N N}(0)$ and $S_{c c}(0)$ are simply related to the mean square number of particles and concentration fluctuations of the system $\left\langle(\Delta N)^{2}\right\rangle$ and $\left\langle(\Delta c)^{2}\right\rangle$. In the case where $\delta=0$, i.e. $\bar{V}_{1}=\bar{V}_{2}$, the fluctuations of the number of particles $\left\langle(\Delta N)^{2}\right\rangle$ is equivalent to the fluctuations of the number density $\left\langle\left(\Delta\left(\begin{array}{l}N \\ V\end{array}\right)\right)^{2}\right\rangle$ if we consider, as we generally do, a constant volume in an open system. These thermodynamic values are directly obtained from normalized small angle scattering experiments [15].

In the general case, $\delta \neq 0$, the Eq. (1) is written as

$$
\begin{aligned}
\frac{1}{N}\left(\frac{\mathrm{d} \sigma}{\mathrm{d} \Omega}\right)_{k=0}^{\mathrm{coh}}= & \langle b\rangle^{2} \frac{N}{V} k_{\mathrm{B}} T K_{T} \\
& +[\langle b\rangle \delta-\langle\Delta b\rangle]^{2} \frac{N k_{\mathrm{B}} T}{\left(\partial^{2} G / \partial c_{2}\right)_{T P N}}
\end{aligned}
$$

or

$$
A K_{T}+B S_{c c}(0) \text {. }
$$

With the approach of a liquid-liquid critical point the first term of this equation is slowly diverging (it is equivalent to the refractive index term in the light scattering experiment) and the second term is strongly diverging, since at $T_{\mathrm{c}}$ the second derivative of the free energy versus concentration tends to zero; but of course the neutron coherent scattering signal will depend also on the two contrasts as expressed in the $B$ coefficient, i.e. $\bar{V}_{1}-\bar{V}_{2}$ or $\delta$ and $b_{1}-b_{2}$ or $\langle\Delta b\rangle$. We recall that the neutron small angle scattering at $k \neq 0$ is, in a first approximation, easily described by standard procedures such as the Ornstein-Zernike relation.

The $B$ coefficient may be written as

$$
B=\left[\frac{\bar{V}_{1} b_{2}-\bar{V}_{2} b_{1}}{c_{1} \bar{V}_{1}+c_{2} \bar{V}_{2}}\right]^{2},
$$

and we obtain a single contrast

$$
B=\left[N \frac{\bar{V}_{1} \bar{V}_{2}}{V}\right]^{2}\left(b_{1}{ }^{\prime}-b_{2}{ }^{\prime}\right)^{2},
$$

where $b_{1}{ }^{\prime}=b_{1} / \bar{V}_{1}$ and $b_{2}{ }^{\prime}=b_{2} / \bar{V}_{2}$ are the scattering lengths per unit volume.

It is this contrast between scattering lengths per unit volume of the two constituants which will determine if a neutron small angle scattering experiment has a chance of a successfull outcome. We must emphasize that even in the case where $b_{1}$ is near $b_{2}$ we might have a strong small angle signal due to the volume differences between the two components. Of course, this raises the question of the correct identification of these two components. In the case of compound formation in a binary mixture we might e.g. encounter concentration fluctuations between one pure component and this compound.

In systems which present a liquid-liquid miscibility gap and where the two extrema of the concentration fluctuations have been well identified, 
the excess volume of mixing is often negligible, i.e. $\bar{V}_{1}$ and $\bar{V}_{2}$ are nearly concentration independent and equal to the volume of the pure components, or more exactly of the two extrema of the fluctuation. Therefore the contrast $b_{1}{ }^{\prime}-b_{2}{ }^{\prime}$ is a characteristic of the system which might depend very slightly on concentration.

Here comes in the interesting effect of isotopic substitution. Ruppersberg [16] has extensively used the negative value of the ${ }^{7}$ Lithium scattering length to investigate alloy concentrations where $\langle b\rangle$ is zero, i.e. [see Eq. (1)] the $S_{c c}$ partial constitutes the whole scattering pattern. In the case of the Li-Na mixture this is particularly interesting since the "zero alloy" concentration is very near the liquid-liquid critical concentration. We might however argue that in the case of atoms of different sizes the concentration $c_{2}$ is probably not the right order parameter, the volume fraction being eventually more appropriate. We may also suggest using isotopic substitution to investigate systems where $b_{1}{ }^{\prime}-b_{2}{ }^{\prime}$ is zero. This would bring the possibility of studying directly the slow divergence of the compressibility term $A K_{T}$ near $T_{\mathrm{c}}$. It would be most interesting to figure out what are the species which take part in the concentration fluctuation process. Indeed $b_{1}{ }^{\prime}$ and $b_{2}{ }^{\prime}$ refer to the actual scattering species which are not necessarily the pure constituents. As seen above, there are cases where complex formation occurs and a miscibility might happen between a pure constituent and a complex; in the system lithium in liquid $\mathrm{ND}_{3}$ for example, there are fluctuations between pure $\mathrm{ND}_{3}$ and the solvated metal $\mathrm{Li}\left(\mathrm{ND}_{3}\right)_{n}$. If by isotopic substitution or concentration changes we succeed in producing a zero contrast $\left(b_{1}{ }^{\prime}-b_{2}{ }^{\prime}=0\right)$ we obtain directly the ratio of the partial molar volume of the two terms of the fluctuation, i.e. informations about the real species involved in the fluctuation process.

\section{A convenient formalism for liquid binary mixture investigations}

We have seen that a significant parameter for small angle concentration fluctuations is the contrast between the scattering lengths per unit volume. This leads us to show how the set of partial structure factors introduced by Bhatia should be corrected in the case of atoms of different size. The scattering experiments on liquid binary mixtures are generally performed on open systems at constant scattering volume defined by the collimated incoming neutron beam on the sample and not at constant number of particles. We are therefore in the condition of a grand canonical ensemble. Under those conditions, Hill [20] has shown that there is no coupling between number density and concentration fluctuations. Bhatia and Thornton however [Eq. (A 7) in [19]] introduce their variables, number of particles and concentration in such a way that the number density might also fluctuate through concentration effects, since in the case of atoms of different sizes there is no explicit volumic constraint on the fluctuations of the number of species 1 and 2 . This is why the $S_{N N}(0)$ term contains not only a compressibility term but also a term depending on the fluctuations of concentration, through the difference in the partial molar volume of the two species. It is however possible to remove this coupling. One may simply, for example, rewrite the scattering Eq. (1) as a function of the fluctuations of the volume fractions instead of the mole fractions. This is a standard alternative of expressing concentrations.

We define the volume fraction

$$
\varphi_{1}=c_{1} \bar{\nabla}_{1} /\left(c_{1} \bar{\nabla}_{1}+c_{2} \bar{\nabla}_{2}\right)
$$

and a scattering volume $V$ defined as

$$
V=N_{1} \bar{\nabla}_{1}+N_{2} \bar{\nabla}_{2} \text {. }
$$

We are now looking for a relation of the type

$$
\begin{aligned}
\frac{1}{V}\left(\frac{\mathrm{d} \sigma}{\mathrm{d} \Omega}\right)^{\mathrm{coh}}= & \left\langle b^{\prime}\right\rangle^{2} S_{V V}(k)+\left\langle\Delta b^{\prime}\right\rangle^{2} S_{\varphi \varphi}(k) \\
& +2\left\langle b^{\prime}\right\rangle\left\langle\Delta b^{\prime}\right\rangle S_{V \varphi}(k)
\end{aligned}
$$

with

$$
\begin{aligned}
\left\langle b^{\prime}\right\rangle & =\varphi_{1} b_{1}{ }^{\prime}+\varphi_{2} b_{2}{ }^{\prime} ; \quad b_{i}{ }^{\prime}=b_{i} / \bar{\nabla}_{i}, \\
\left\langle\Delta b^{\prime}\right\rangle & =b_{1}{ }^{\prime}-b_{2}{ }^{\prime}
\end{aligned}
$$

and with $S_{V V}$ and $S_{\varphi \varphi}$ referring to the total volume and the volume fraction fluctuations.

In the $k \rightarrow 0$ limit the relation between the new set of thermodynamic variables and the previous one is

$$
\left(\frac{\partial^{2} G}{\partial \varphi_{2}^{2}}\right)_{T P N}=\left(\frac{\partial^{2} G}{\partial c_{2}^{2}}\right)_{T P N} \frac{\left(c_{1} \bar{\nabla}_{1}+c_{2} \bar{\nabla}_{2}\right)^{3}}{\left(\bar{\nabla}_{1} \bar{\nabla}_{2}\right)^{2}}
$$

and therefore

$$
S_{\varphi \varphi}(0)=\frac{N}{V}\left(\frac{N}{V} \bar{\nabla}_{1} \bar{\nabla}_{2}\right)^{2} S_{c c}(0) .
$$


In the same way

$$
S_{V V}(0)=k_{\mathrm{B}} T \cdot K_{T}
$$

The relations (6) and (3) with (5) can now be identified as a proof that there is no coupling between $V$ and $\varphi$ and thus $S_{V \varphi}(0)=0$. We therefore obtain for the scattering at $k=0$

$$
\frac{1}{V}\left(\frac{\mathrm{d} \sigma}{\mathrm{d} \Omega}\right)_{k=0}^{\mathrm{coh}}=\left\langle b^{\prime}\right\rangle^{2} S_{V V}(0)+\left\langle\Delta b^{\prime}\right\rangle^{2} S_{\varphi \varphi}(0) .
$$

The first term of this equation is actually the total number density fluctuation $\left\langle(\Delta \varrho)^{2}\right\rangle / \varrho^{2}$ where $\varrho=N /\langle V\rangle$.

This result has been achieved because the local deviation $\Delta \varphi$ from the mean volume fraction $\varphi$, which is

$$
V \Delta \varphi=\varphi_{2} \bar{V}_{1} \Delta N_{1}-\varphi_{1} \bar{V}_{2} \Delta N_{2},
$$

becomes, at fixed volume $V$, entirely symmetric with respect to fluctuations of the number of species 1 or 2 , even for species of different sizes. The fixed volume $V$ condition implies that

$$
\bar{V}_{1} \Delta N_{1}=-\bar{V}_{2} \Delta N_{2} \text {. }
$$

There is no such a condition in the Bhatia and Thornton formalism, where the local deviation from the mean concentration $c$, at fixed number of particles $N$,

$$
N \Delta c=c_{2} \Delta N_{1}-c_{1} \Delta N_{2}
$$

has asymmetric volume effects and consequently raises differences between fluctuations of number of particles and of number density. If one simply adds the condition (10), i.e. the requirement that the fluctuations of concentration do not lead to any change in volume, to the Bhatia and Thornton formalism, one however decouples also effectively the number density fluctuations from the concentration fluctuations.

The Eqs. (6), (7) and (8) present therefore an adequate set of partial structure factors in the small angle scattering region and at the approach of the thermodynamic limit, since each partial is clearly related to one of the two identified physical fluctuations.

For large values of $k$, the interest of the formalism is less obvious. $S_{V \varphi}(k)$ does not tent to zero for large $k$ values as does $S_{N c}(k)$ [19]. It is however quite plausible that $S_{V \varphi}(k)$ structurless as it has been assumed for $S_{N c}(k)$. This question is very important for the experimental determination of the partical structure faktors since it would allow to reduce the problem to the determination of only two partial the structure factors instead of three, and this in the general case where the constituants have different sizes.

\section{IV. $S_{c c}(0)$ or $S_{\varphi \varphi}(0)$ and the distribution of the concentration fluctuations}

If the fluctuations are not too large, $S_{c c}(0)$ is directly related to the mean square concentration fluctuation; $\left\langle(\Delta c)^{2}\right\rangle=\left\langle(c-\bar{c})^{2}\right\rangle$ with $\bar{c}$ the mean concentration, i.e.

$$
S_{c c}(0)=N k_{\mathrm{B}} T /\left(\partial^{2} G / \partial c^{2}\right)_{T P N}=N\left\langle(\Delta c)^{2}\right\rangle .
$$

In a critical region this relationship does not tell the whole story about the fluctuations since the distribution of concentrations about the mean has a strongly skewed Gaussian shape [17]. Higher momenta should therefore be investigated to describe carefully the concentration distribution. Nevertheless the second momentum, which gives the width of the Gaussian distribution, is always the leading term and is generally sufficient to describe the overall feature of the fluctuations.

The problem is now to define a relevant volume scale in which the concentration fiuctuation distribution can be studied. If $V$ is the volume of the vessel, $N$ is a very large number indeed, and for a given $S_{c c}(0),\left\langle(\Delta c)^{2}\right\rangle$ always tends to zero; the distribution of concentration at the volume scale of the whole sample is actually a $\delta$ function. The volume scale for fluctuations is certainly smaller, however, and must be related to the coherence length $\xi$ of the fluctuations as measured in the neutron scattering experiment. Thus the relevant $N$ in this volume must be proportional to $\xi^{3}$ in (9). It is only at this $\xi^{3}$ volume scale that the distribution of concentrations is observable.

It is now easy to find out the behaviour of $\left\langle(\Delta c)^{2}\right\rangle$ in the vicinity of a critical point. We choose $\bar{c}$ as the critical concentration for the sake of simplicity. $S_{c c}(0)$ is known to diverge [18] when $T-T_{\mathrm{c}}$ goes to zero along the critical isochore with a power law of the type

$$
S_{c c}(0)=C\left(\frac{T-T_{\mathrm{c}}}{T_{\mathrm{c}}}\right)^{-\gamma} .
$$


In the same way $\xi$ diverges as

$$
\xi=\xi_{0}\left(\frac{T-T_{\mathrm{c}}}{T_{\mathrm{c}}}\right)^{-v},
$$

where $\gamma$ and $v$ are critical exponents whose values are

$$
1<\gamma<1.30, \quad 0.5<v<0.66 \text {. }
$$

Thus $\left\langle(\Delta c)^{2}\right\rangle$ at a temperature dependent $\xi^{3}$ volume scale follows the law

$$
\left\langle(\Delta c)^{2}\right\rangle=c^{\prime}\left(\frac{T-T_{\mathrm{c}}}{T_{\mathrm{c}}}\right)^{3 v-\gamma},
$$

and since $\gamma=v(2-\eta)[18]$ we obtain

$$
\left\langle(\Delta c)^{2}\right\rangle=C^{\prime}\left(\frac{T-T_{\mathrm{c}}}{T_{\mathrm{c}}}\right)^{r(1-\eta)},
$$

where $\eta$ is an exponent whose value is $0<\eta<0.10$. It is interesting to emphasize the fact that, as $T$

[1] W. Schmatz, T. Springer, J. Schelten, and K. Ibel, J. Appl. Cryst. 7, 96 (1974).

[2] P. C. Hohenberg in "Critical Phenomena", proceedings of the international school of Physics "Enrico Fermi" course LI edited by M. S. Green, Academic Press, New York 1971, p. 300.

[3] J. Goulon, M. Hollecker, J. M. Thiebaut, and D. Oxtoby, to be published.

[4] M. J. Sienko and P. Chieux in "Metal-Ammonia Solutions", proceedings of Colloque Weyl II, editors J. J. Lagowski and M. J. Sienko, Butterworths, London 1970 , p. 339.

[5] N. F. Mott, Metal-Insulator Transitions, Taylor and Francis, London 1974

[6] J. F. Jal, G. Guiraud, and P. Chieux, J. Dupuy, J. Phys. E 10, 977 (1977).

[7] P. Chieux, J. Phys. Chem. 79, 2891 (1975).

[8] J. F. Jal, P. Chieux, und J. Dupuy, Ber. Bunsenges. Phys. Chem. 80, 820 (1976).

[9] V. P. Warkulwiz, B. Mozer, and M. S. Green, Phys. Rev. Lett. 32, 1410 (1974).

[10] P. Chieux, Phys. Lett. A 48 (6), 493 (1974). goes to $T_{c}$ the width of the distribution of concentrations actually decreases and vanishes at $T_{\mathrm{c}}$. This is due to the fact that even if $S_{c c}(0)$ diverges, making the fluctuations easier to occur, the number of molecules $N$ involved in a correlated fluctuation diverges faster. In the vicinity of the critical point, the spatial extent of the fluctuations becomes indeed very large due to the divergency of $\xi$ but their distribution is actually damped and vanishes at $T_{\mathrm{c}}$. At a temperature very near $T_{\mathrm{c}}$ the system fluctuates between two macroscopic phases with nearly the same concentration [17].

\section{Acknowledgements}

One would like to thank H. Ruppersberg and P. Desré for numerous discussions, P. Bergé for his kind comments on light scattering conditions, and J. Blètry for his computer simulations of $S_{V \varphi}(k)$.

[11] J. F. Jal, P. Chieux, and J. Dupuy, J. Appl. Cryst. 11, $58(1978)$.

[12] H. Ruppersberg and W. Knoll, Z. Naturforsch. 32a, 1374 (1977).

[13] E. S. Wu and H. Brumberger, Phys. Lett., 53 A, 475 (1975).

[14] M. Weber, S. Steeb, and W. Knoll, Phys. Lett. 61 A, 78 (1977).

[15] P. Chieux and P. Damay, Chem. Phys. Lett., to be published.

[16] H. Ruppersberg and H. Egger, J. Chem. Phys. 63, 4095 (1975).

[17] P. Damay and P. Schettler, J. Phys. Chem. 79 (26), 2930 (1975).

[18] M. E. Fisher, J. Math. Phys. 5 (7) 944 (1964); M. E. Fisher and A. Aharony, Phys. Rev. B10 (7) 2818 (1974).

[19] A. B. Bhatia and D. E. Thornton, Phys. Rev. B2, 3004 (1970).

[20] T. H. Hill, Statistical Mechanics, McGraw Hill, New York 1956, p. 113. 